\section{Case Reports in Ophthalmology}

Case Rep Ophthalmol 2021;12:48-56

DOI: 10.1159/000510330

Published online: January 13, 2021 (c) 2021 The Author(s)

Published by S. Karger AG, Basel www.karger.com/cop

\title{
Efficacy of Psychiatric Treatment to Treat a Specific Phobia of Intravitreal Injections in a Patient with Neovascular Age-Related Macular Degeneration
}

\author{
Atsuta Ozaki ${ }^{a}$ Hisashi Matsubara ${ }^{a}$ Masahiko Sugimoto ${ }^{a}$ \\ Manami Kuze $^{b} \quad$ Mineo Kondo $^{a} \quad$ Takashi Shiroyama $^{c}$ \\ aDepartment of Ophthalmology, Mie University Graduate School of Medicine, Tsu, Japan; \\ bDepartment of Ophthalmology, Matsusakachuo General Hospital, Matsusaka, Japan; \\ 'Department of Neuropsychiatry, Mie University Graduate School of Medicine, Tsu, Japan
}

\section{Keywords}

Specific phobia $\cdot$ Intravitreal injection $\cdot$ Psychiatric treatment $\cdot$ Retinal angiomatous proliferation · Cognitive-behavioral therapy

\begin{abstract}
Intravitreal injection of anti-vascular endothelial growth factor (anti-VEGF) is essential for the treatment of macular diseases such as wet age-related macular degeneration and macular edema. Although continued treatment is needed to maintain good vision, some patients cannot continue such injections for various reasons, including specific phobias. Here, we report a case of a patient with a specific phobia of intravitreal injections who could resume treatment after undergoing combined drug and cognitive-behavioral therapy (CBT). A 74-year-old Japanese man diagnosed with retinal angiomatous proliferation by fluorescein angiography and indocyanine green angiography was treated with intravitreal anti-VEGF injection. However, at 8 months after the first treatment, he became difficult to treat because of a phobia of injections. He was treated with photodynamic therapy, but his macular edema did not improve. After a psychiatric consultation, he was diagnosed with a specific phobia of intravitreal injections. Combined drug and $C B T$ enabled him to resume receiving intravitreal injections. This
\end{abstract}

\begin{tabular}{ll}
\hline & Atsuta Ozaki \\
Department of Ophthalmology & Mie University Graduate School of Medicine \\
& 2174 Edobashi, Tsu City, Mie 514-8507 (Japan) \\
atsuta3527@gmail.com
\end{tabular}




\section{Case Reports in Ophthalmology}

Case Rep Ophthalmol 2021;12:48-56

DOI: $10.1159 / 000510330$

(c) 2021 The Author(s). Published by S. Karger AG, Basel www.karger.com/cop

Ozaki et al.: Psychiatric Treatment for a Specific Phobia of Intravitreal Injections

case demonstrates that a specific phobia of intravitreal injections may benefit from combined drug and CBT. In this regard, some patients with high anxiety and fear of intravitreal injections should be referred to a psychiatrist at an early stage.

(C) 2021 The Author(s)

Published by S. Karger AG, Basel

\section{Introduction}

Intravitreal injection of anti-vascular endothelial growth factor (anti-VEGF) is used to treat patients with neovascular age-related macular degeneration (nAMD) and macular edema (ME) and improve their visual function. Therefore, this treatment is necessary for a variety of macular diseases.

However, intravitreal injections must be continued to maintain good vision [1]. Especially in patients with nAMD, visual acuity decreases with insufficient treatment [1], highlighting the importance of a proactive treatment regimen. In particular, retinal angiomatous proliferation (RAP) is a special type of nAMD that has been reported to require frequent treatment with anti-VEGF [2]. However, for various reasons, some patients cannot continue to receive drug injections. Here, we report a case who could not continue treatment because of a specific phobia of intravitreal injections but responded well to psychiatric treatment.

\section{Case Report}

The present case was a 74-year-old Japanese man with an unremarkable medical history who complained of visual disorders in the left eye. He was diagnosed with ME in the left eye and referred to our hospital. At the initial examination, his decimal best-corrected visual acuity (BCVA) based on a visual acuity chart was 0.9 in his right eye and 1.2 in his left. Intraocular pressure was $13 \mathrm{~mm} \mathrm{Hg}$ in both eyes. Slit-lamp examination showed no abnormalities except for mild cataracts in both eyes. Ophthalmoscopy showed multiple soft drusen around the fovea in both eyes (Fig. 1a, b). Spectral-domain optical coherence tomography (SD-OCT) showed pigment epithelial detachment and ME in his left eye (Fig. 1c). Fluorescein angiography showed leakage from choroidal neovascularization (CNV) and retinal-retinal anastomosis in the early phase (Fig. 1d). Indocyanine green angiography showed a "hot spot," which was found to be leakage from CNV in the late phase (Fig. 1e). Based on these findings, we made a diagnosis of RAP.

We started treatment with intravitreal ranibizumab (IVR) injections once a month for 3 months, after which, SD-OCT showed improved ME. At that time, the BCVA in his left eye was 0.7. Although he agreed to receive the third intravitreal injection, he expressed his fear of it. After the third injection, a fixed-dosed protocol every 8 weeks was selected. At the fourth injection, he was aware of itching and moved his body involuntarily after an eyelid opener was applied. As a result, we considered that giving the intravitreal injection was dangerous, so we decided to skip the injection at that time. However, 4 weeks later, we attempted again, and he received the IVR injection without any problems. After another 4 weeks, SD-OCT showed ME in his left eye, so we switched from ranibizumab to aflibercept. Subsequently, we performed three additional successful intravitreal aflibercept (IVA) injections. At 8 weeks from last IVA injection, SD-OCT showed recurrent ME in his left eye. At the time of the fourth IVA injection, he reported severe itchiness immediately after the eyelid opener was applied, which made it

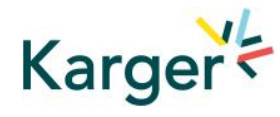


difficult to give the injection, so we skipped it again. However, after 3 days, it was possible to give the IVA injection as he reported only slight itchiness. Because of the deterioration of the ME, we shortened the period of the fixed-dose protocol from 8 to 6 weeks. The fifth and sixth IVA injections were successful despite his pain and movement. However, in accordance with his request, we changed the treatment from a fixed-dose to an as-needed protocol. The BCVA of his left eye at that time was 0.7 .

At 7 months from the last administration, we decided to give another IVA injection because of recurrent ME. However, it was impossible to perform the injection because the patient complained of severe facial pain due to a fear of intravitreal injections. We then judged that subsequent treatment by intravitreal injection would be difficult. Therefore, as an alternative, we began treatment with photodynamic (PDT) monotherapy three times. As a result, his ME showed no improvement on SD-OCT (Fig. 2a-d).

Accordingly, he was referred to a psychiatrist in the hopes of continuing treatment with intravitreal injections. He was diagnosed with a specific phobia of intravitreal injections based on his medical history from our Department. We started treatment for specific phobia 11 months after the recurrence. He began escitalopram ( $5 \mathrm{mg}$ ) regularly and bromazepam (5 mg) 30 min before the injection. Additionally, as cognitive-behavioral therapy (CBT), we conducted a total of six intravitreal injection simulations twice a week. The following was performed as the simulations. We performed a procedure similar to intravitreal injections except for sticking an injection into the eye after proclaiming that I will not inject today. If the patient wanted to discontinue because of the phobia, it was discontinued. The simulations reduced his itching, movement, and anxiety at the time of the preparation for the intravitreal injections.

As a result, the psychiatric treatment enabled him to get receive the intravitreal injections without any problems 3 days after the sixth simulation. The psychiatric treatment was continued, and 1 month later, the patient underwent combined IVA and PDT. His BCVA after treatment was 0.2. At present, no change in effusion has been observed (Fig. 3), and the patient is continuing with the as-needed follow-up protocol.

\section{Discussion}

Specific phobia is classified as an anxiety disorder in the Diagnostic and Statistical Manual of Mental Disorders, Fifth Edition. Specific phobia causes fear and anxiety in specific situations or targets, and includes symptoms such as anticipatory anxiety, autonomic nervous symptoms, and physical and mental tension. The main problem with specific phobia involves attempts to avoid the object of fear. If the object of fear is medical practice, treating the patient becomes difficult and the medical condition worsens. The treatment of specific phobia is divided into drug therapy and CBT. These treatments are recommended for patients without dementia or intellectual disability who make an effort to be treated.

Drug therapy for specific phobia is similar to that for anxiety. We used selective serotonin reuptake inhibitors (SSRIs) regularly and benzodiazepines before the injection. SSRIs exert beneficial effects on the mental symptoms of phobia and have been reported to reduce anticipatory anxiety in fearful situations $[3,4]$. The advantage of SSRIs is that they are not considered to cause dependence or cognitive impairment. By contrast, the disadvantage of SSRIs is that 2-6 weeks of treatment is needed to achieve the desired effect. Benzodiazepines are effective for the physical symptoms of phobia, and also exert a muscle relaxant action [3, 4]. The advantages of benzodiazepines are that they have an immediate effect and reduce physical

\section{Karger'=}




\section{Case Reports in Ophthalmology}

symptoms immediately before a fearful situation. By contrast, the disadvantages of benzodiazepines are dependence and the possibility of a rebound phenomenon.

Evidence-based treatment includes exposure therapy in CBT for specific phobia [5]. Exposure therapy aims to enable patients to eliminate anxiety and fear by exposing them to the fearful situation. Exposure therapy for a specific phobia is a similar simulation of the actual procedure. We perform a procedure similar to intravitreal injections except for sticking an injection. It is important to state that we do not inject into the eye to eliminate anxiety. It is necessary to continue the simulation because of uneven anxiety even if the exposure therapy is effective and the intravitreal injection is successful. The advantages of exposure therapy include limited physical side effects, easy motivation for treatment, a low recurrence rate, and easy standardization.

RAP is classified as a type of nAMD [6]. RAP has been reported to affect the fellow eye of all cases within 3 years after onset [7]. In recent years, numerous reports have recommended anti-VEGF drug monotherapy for the treatment of RAP. However, frequent vitreous injections are required to maintain and improve visual acuity [2]. On the other hand, PDT monotherapy has been reported to be insufficient to treat RAP [8, 9], while combined IVR and PDT for RAP has been shown to be effective for helping patients improve and maintain visual acuity with only a small number of treatments [10]. However, no consensus has been reached regarding the optimal treatment for RAP. As is evident from past reports, maintaining treatment using anti-VEGF drug therapy is important for treating RAP. Moreover, the possibility of developing RAP in both eyes must be considered.

In this case, the CBT and drug therapy were remarkably effective. The patient's fear and anxiety about the intravitreal injections were reduced, and they could be resumed without fear. we started escitalopram (5 $\mathrm{mg}$ ) regularly and bromazepam (5 $\mathrm{mg}$ ) $30 \mathrm{~min}$ before the injection. It took 7 weeks to give an intravitreal injection from the start of drug treatment. However, due to the ineffectiveness of PDT monotherapy and the continued exudation, the patient's BCVA after combined IVR and PDT was 0.2. The intravitreal injections could be resumed for about 2 months after the start of psychiatric treatment. If the patient had been referred immediately after the recurrence of the exudation, we may have been able to reduce the duration of the treatment interruption. On the other hand, we also considered combining the intravitreal injection with intravenous or inhalation sedation with nitrous oxide anesthesia. However, as we perform intravitreal injections frequently in the outpatient clinic, it was not possible in this case because of limits in the hospital equipment, physician management skills with regard to sedation, respiratory depression, and air pollution. Furthermore, RAP often affects both eyes, so in this case, frequent intravitreal injections will likely be required. If frequent vitreous injections accompanied by intravenous or inhalation sedation are required, doctors and patients will face greater burden. By contrast, in this case, psychiatric treatment was considered useful for treating the specific phobia because it was very similar to the usual injection technique and did not require any special techniques or equipment.

\section{Conclusion}

In conclusion, a patient diagnosed with a specific phobia of intravitreal injections could resume anti-VEGF treatment as a result of psychiatric treatment. As with this case, some patients cannot continue intravitreal injections due to a specific phobia, and such patients may be undiagnosed in actual clinical practice. For patients suspected of having a specific phobia,

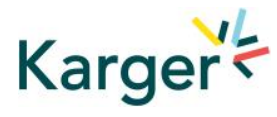




\section{Case Reports in Ophthalmology}

Ozaki et al.: Psychiatric Treatment for a Specific Phobia of Intravitreal Injections

early interviews and psychiatric interventions may be successful. As this is only a case report, it does not address individual differences in drug therapy and CBT. Therefore, more patients need to be treated in the future, and individualized treatment for a specific phobia of intravitreal injections needs to be considered.

\section{Statement of Ethics}

The patient provided written informed consent for submission of the case report and any accompanying images. This study was approved by the Ethics Committee of Mie University Graduate School of Medicine, Tsu City, Mie, Japan.

\section{Conflict of Interest Statement}

The authors have no conflicts of interest to declare.

\section{Funding Sources}

There was no funding source for this study.

\section{Author Contributions}

Atsuta Ozaki wrote the initial draft of the manuscript. Atsuta Ozaki and Hisashi Matsubara contributed to conception of the study, design, and data analysis. All authors revised the manuscript critically for important intellectual content and have given final approval of the version to be published and agreed to be accountable.

\section{References}

1 Rofagha S, Bhisitkul RB, Boyer DS, Sadda SR, Zhang K; SEVEN-UP Study Group. Seven-year outcomes in ranibizumab-treated patients in ANCHOR, MARINA, and HORIZON: a multicenter cohort study (SEVEN-UP). Ophthalmology. 2013 Nov;120(11):2292-9.

2 Engelbert M, Zweifel SA, Freund KB. "Treat and extend" dosing of intravitreal antivascular endothelial growth factor therapy for type 3 neovascularization/retinal angiomatous proliferation. Retina. 2009 NovDec;29(10):1424-31.

3 Rickels K, Schweizer E. The clinical course and long-term management of generalized anxiety disorder. J Clin Psychopharmacol. 1990 Jun;10(3 Suppl):101S-10S.

4 Baldwin DS, Anderson IM, Nutt DJ, Bandelow B, Bond A, Davidson JR, et al.; British Association for Psychopharmacology. Evidence-based guidelines for the pharmacological treatment of anxiety disorders: recommendations from the British Association for Psychopharmacology. J Psychopharmacol. 2005 Nov;19(6):567-96.

5 Barlow DH, Lehman CL. Advances in the psychosocial treatment of anxiety disorders. Implications for national health care. Arch Gen Psychiatry. 1996 Aug;53(8):727-35.

6 Yannuzzi LA, Negrão S, Iida T, Carvalho C, Rodriguez-Coleman H, Slakter J, et al. Retinal angiomatous proliferation in age-related macular degeneration. Retina. 2001;21(5):416-34.

7 Gross NE, Aizman A, Brucker A, Klancnik JM Jr, Yannuzzi LA. Nature and risk of neovascularization in the fellow eye of patients with unilateral retinal angiomatous proliferation. Retina. 2005 Sep;25(6):713-8.

\section{Karger'=}


8 Boscia F, Furino C, Sborgia L, Reibaldi M, Sborgia C. Photodynamic therapy for retinal angiomatous proliferations and pigment epithelium detachment. Am J Ophthalmol. 2004 Dec;138(6):1077-9.

9 Silva RM, Cachulo ML, Figueira J, de Abreu JR, Cunha-Vaz JG. Chorioretinal anastomosis and photodynamic therapy: a two-year follow-up study. Graefes Arch Clin Exp Ophthalmol. 2007 Aug;245(8):1131-9.

10 Saito M, Iida T, Kano M. Combined intravitreal ranibizumab and photodynamic therapy for retinal angiomatous proliferation. Am J Ophthalmol. 2012 Mar;153(3):504-514.e1. 


\section{Case Reports in Ophthalmology}
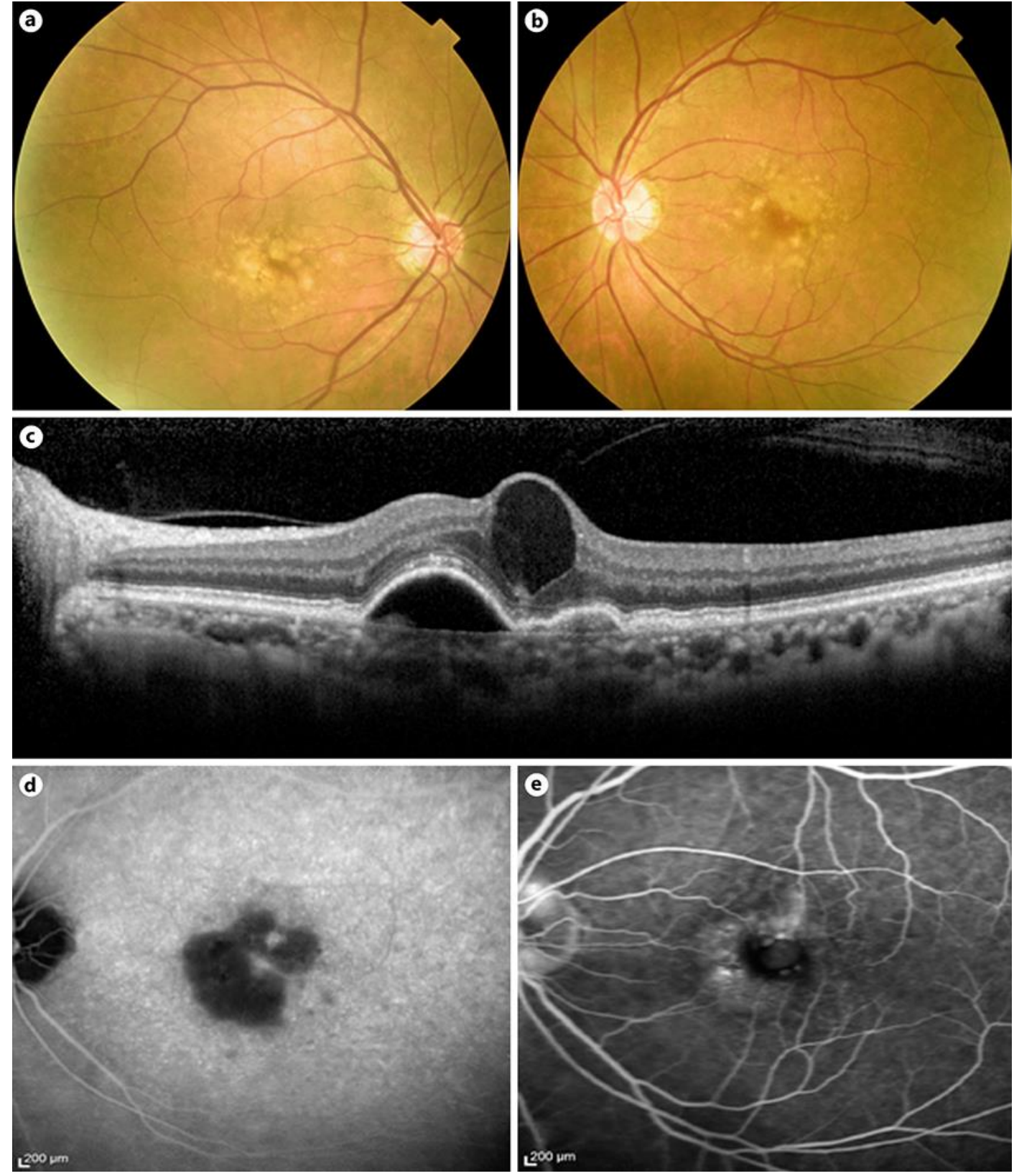

Fig. 1. Fundus photographs of (a) the right eye and (b) left eye; (c) spectral-domain optical coherence tomography (SD-OCT) images of the left eye, and (d) fluorescein angiography (FA) and (e) indocyanine green angiography (ICGA) images of the left eye with RAP at the initial visit. a Multiple soft drusen can be seen around the fovea. b Multiple soft drusen and pigment epithelial detachment (PED) and macular edema (ME) can be seen around the fovea. $\mathbf{c}$ SD-OCT images show PED and ME. $\mathbf{d}$ Early-phase FA image shows leakage from choroidal neovascularization and retinal-retinal anastomosis. e Late-phase ICGA image shows a hyperfluorescent spot above the fovea. 
Case Reports in Ophthalmology
Case Rep Ophthalmol 2021;12:48-56

DOI: $10.1159 / 000510330$

(c) 2021 The Author(s). Published by S. Karger AG, Basel www.karger.com/cop

Ozaki et al.: Psychiatric Treatment for a Specific Phobia of Intravitreal Injections
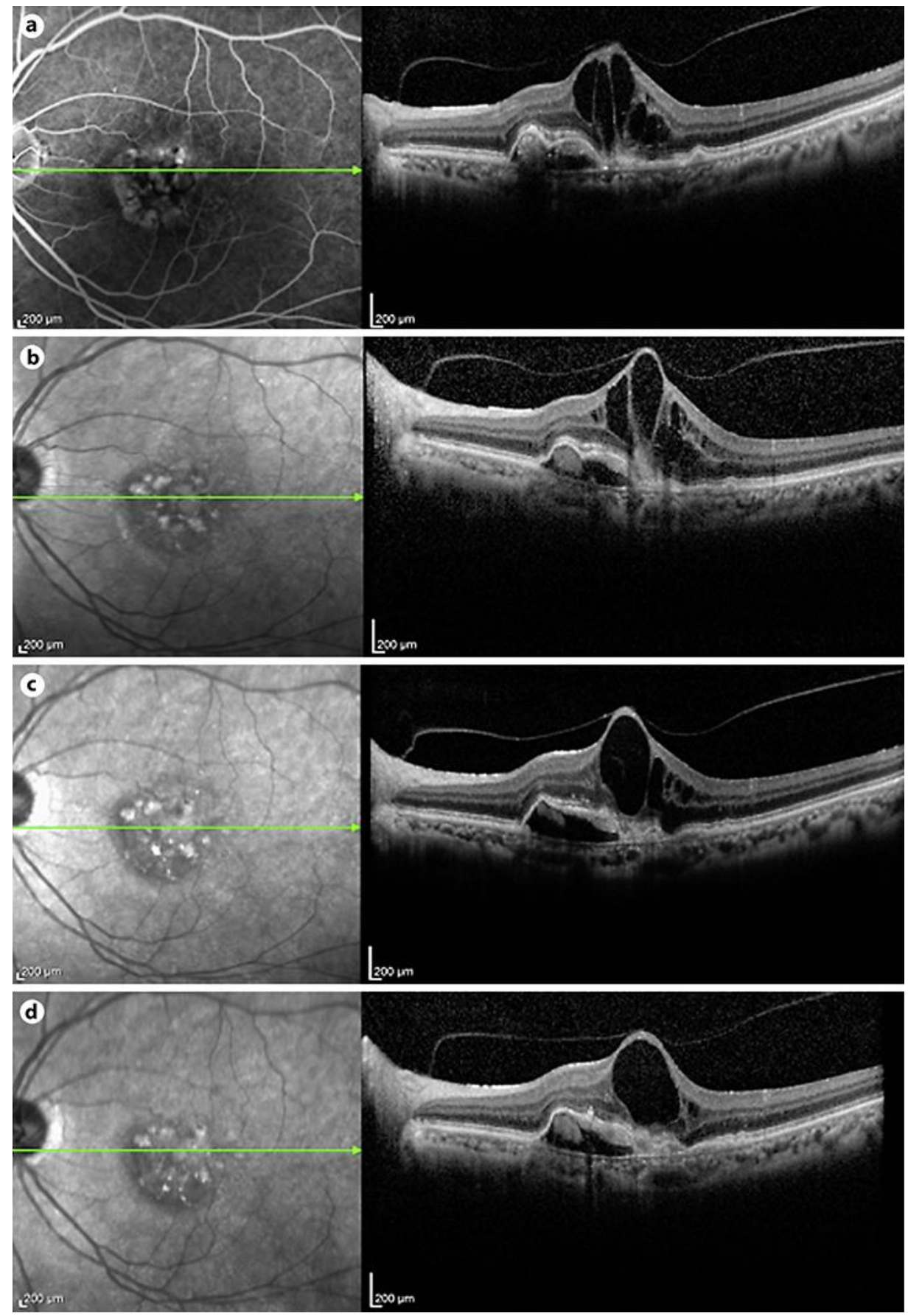

Fig. 2. Spectral-domain optical coherence tomography (SD-OCT) images of the left eye. a SD-OCT image before photodynamic therapy (PDT). b SD-OCT image after the first PDT. c SD-OCT image after the second PDT. $\mathbf{d}$ SD-OCT image after the third PDT. No improvement is seen in exudative lesions from a to d. 
Case Reports in Ophthalmology
Case Rep Ophthalmol 2021;12:48-56 DOI: $10.1159 / 000510330$

(c) 2021 The Author(s). Published by S. Karger AG, Basel www.karger.com/cop

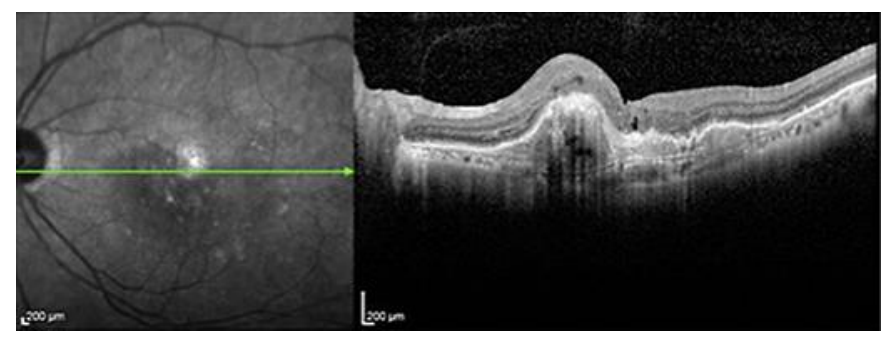

Fig. 3. Spectral-domain optical coherence tomography images of the left eye after treatment with combined intravitreal aflibercept injections and photodynamic therapy. The macular edema is improved. 